\title{
Nanoscale
}

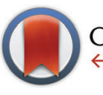

CrossMark \& click for updates

Cite this: Nanoscale, 2016, 8, 8966

Received 7th December 2015,

Accepted 22nd March 2016

DOI: $10.1039 / c 5 n r 08709 k$

www.rsc.org/nanoscale

\section{Carbene-mediated self-assembly of diamondoids on metal surfaces}

\author{
Bibek Adhikari, ${ }^{a}$ Sheng Meng ${ }^{\mathrm{b}}$ and Maria Fyta*a
}

$\mathrm{N}$-heterocyclic carbenes ( $\mathrm{NHC})$ s are emerging as an alternative class of molecules to thiol-based selfassembled monolayers (SAMs), making carbene-based SAMs much more stable under harsh environmental conditions. In this work, we have functionalized tiny diamondoids using NHCs in order to prepare highly stable carbene-mediated diamondoid SAMs on metal substrates. Using quantum-mechanical simulations and two different configurations for the carbene-functionalized diamondoids attached on gold, silver, and platinum surfaces we were able to study in detail these materials. Specifically, we focus on the binding characteristics, stability, and adsorption of the NHC-mediated diamondoid SAMs on the metal surfaces. A preferential binding to platinum surfaces was found, while a modulation of the work function in all cases was clear. The surface morphology of all NHC-based diamondoid SAMs was revealed through simulated STM images, which show characteristic features for each surface.

\section{Introduction}

Diamondoids are tiny hydrogen terminated nanostructures, which are built up by a certain number of diamond-like cages. ${ }^{1,2}$ These nano-sized diamonds can be naturally found in petroleum $^{2}$ and can be organically synthesized ${ }^{3}$ or nucleated from energetic species. ${ }^{4}$ Diamondoids have shown a great potential for novel nanotechnological applications due to their diversity in size and the variety of chemical modifications they can assume. ${ }^{5-7}$ They are also expected to play a significant role as building blocks for functional nanostructures. ${ }^{8}$ The first member of the diamondoid family is adamantane $\mathrm{C}_{10} \mathrm{H}_{16}$. Lower diamondoids, that is the ones that are smaller in size and their derivatives have been theoretically observed to selfassemble into larger inter-linked nanostructures. ${ }^{9}$ A selfassembly of diamondoids encapsulated inside carbon nanotubes was also shown to be possible. ${ }^{10-12}$ These nanometersized cages can attach on metallic surfaces through a thiol group $^{13,14}$ to form self-assembled monolayers (SAMs) with a negative electron affinity ${ }^{5,15}$ and a strong monochromatic emission. ${ }^{5,16}$ These properties make diamondoids very promising for electronics applications. In the past, thiol based SAMs on metal surfaces have led to significant applications in the field of surface emission, sensing, electrochemistry, drug delivery, and microelectronics. ${ }^{17-21}$ However, the thermal instability of these thiol-based SAMs on gold under harsh environmental

\footnotetext{
${ }^{a}$ Institute for Computational Physics, Universität Stuttgart, Allmandring 3, 70569 Stuttgart, Germany. E-mail: mfyta@icp.uni-stuttgart.de

${ }^{b}$ Institute of Physics, Chinese Academy of Sciences, Zhongguancun, Beijing, 100190,
} China conditions has been a huge hindrance in using them for industrial purposes. ${ }^{22-26}$ Thiol-based SAMs are found to be stable only when stored in a ultra-high vacuum in the absence of light, but tend to degrade after a few weeks at room temperature. ${ }^{27-29}$

Accordingly, an alternative molecule which mediates the formation of diamondoid SAMs on substrates is needed. For this, we propose the use of N-heterocyclic carbene (NHC) molecules to promote the binding of diamondoids on metal surfaces. Unlike typical carbene molecules, which are highly reactive and have very limited stability, NHCs usually have two hetero-atoms adjacent to the carbene carbon..$^{30}$ This feature increases the NHC stability. In addition, NHCs can be prepared on the industrial scale, can be crystallized, distilled, and stored for long periods of time in order to become commercially available reagents. ${ }^{31-33}$ Bulky NHC ligands have also theoretically been shown to stabilize main group compounds. ${ }^{34}$ In this work, we aim to study the structural and electronic properties of carbenefunctionalized diamondoid monolayers on metal surfaces. For the latter, we consider gold, silver, and platinum surfaces. We study the structural and electronic properties of the diamondoid SAMs on these surfaces, their morphology, and work function in view of their nanotechnological applications as potential electron emitters and sensors. This paper is structured as follows: in the next section we present the methodology and materials used for this investigation and move on to the analysis of the results of adsorption characteristics, electronic properties and surface morphology. The conclusions of this work are summarized in the final section. 


\section{Methods}

Density functional theory (DFT) based calculations were performed using the Vienna $a b$ initio simulation package (VASP). ${ }^{35}$ Exchange correlation at the level of generalized gradient approximation (GGA) ${ }^{36}$ using the PW91 exchange-correlation functional for the electrons ${ }^{37,38}$ was implemented. A projector augmented wave (PAW) basis was applied ${ }^{36,37}$ and the wave functions were expanded in a plane-wave basis set with a kinetic energy cutoff of $400 \mathrm{eV}$ for all the systems studied here. The optimized lattice constants of the metal substrate were obtained through structural optimization. The metal (111) surfaces were modeled using slabs of four atomic layers. It was tested in advance that this number of layers is sufficient to reproduce the surface-like properties of the metals. The vacuum layers for the surfaces were also chosen to be thick enough to ensure decoupling between neighbouring slabs. During relaxation, atoms in the lower two atomic layers were fixed in their respective bulk positions, and all the other atoms were allowed to relax including the molecules on the surface. A gamma-centered $3 \times 3 \times 1 k$-point mesh was used for the $5 \times 5$ surface unit cell of metals. In the present study, all the calculations are non-spin-polarized. For the structural relaxation the Methfessel-Paxton scheme was used with a smearing parameter of $0.2 \mathrm{eV} \cdot{ }^{39,40}$ The total energy of a relaxed structure was calculated again using the tetrahedron method. ${ }^{41}$ The metal surfaces we have considered here are all (111) surfaces of $\mathrm{Au}, \mathrm{Ag}$, and $\mathrm{Pt}$, and are a representative class of metallic surfaces used in experiments and electronics applications. According to our calculations, the optimized lattice constants for the bulk form of $\mathrm{Au}, \mathrm{Ag}$ and Pt were found to be
$4.175 \AA$ A $4.160 \AA$, and $3.985 \AA$, respectively. These are in a very good agreement within $2-3 \%$ with the experimental values $4.065 \AA$ А $4.079 \AA$ A, and $3.912 \AA$, respectively. ${ }^{42}$

\section{Results and discussion}

The smallest diamondoid adamantane $\mathrm{C}_{10} \mathrm{H}_{16}$ was chosen to model a free radical self-assembled monolayer absorbed on the metal substrates. Two variations of carbene molecules were chosen and attached to adamantane to prepare two different diamondoid SAMs, namely $\mathrm{C}_{13} \mathrm{~N}_{2} \mathrm{H}_{18}$ and $\mathrm{C}_{23} \mathrm{O}_{1} \mathrm{~N}_{2} \mathrm{H}_{32} \cdot{ }^{43}$ We refer to these in the following sections as NHC1 and NHC2, respectively. The former is chosen on a pure theoretical basis, while the latter has already been used in experiments. We chose both as representative carbene candidates in order to extend the search for potential carbene-mediated diamondoidbased SAMs. $\mathrm{C}_{23} \mathrm{O}_{1} \mathrm{~N}_{2} \mathrm{H}_{32}$ has been investigated experimentally and $\mathrm{C}_{13} \mathrm{~N}_{2} \mathrm{H}_{18}$ is a molecule with less complexity and could be easier to manipulate. Before structural relaxation, the NHCmediated diamondoid-SAMs were placed on the on-top site of the surfaces and at a distance larger than $2.20 \AA$ A. Preferential site binding of the NHC2 on the surfaces is not considered in this work taking into account experimental evidence that the lowest carbene-C atom (for NHC2) is bonded on the on-top site on a $\mathrm{Au}(111)$ surface. $^{43}$ Our initial calculations also showed that the on top site is also preferred by the NHC1mediated adamantane.

Two different molecules chosen to self-assemble on the metal surfaces are shown in Fig. 1. The figure shows adamantane functionalized with the carbene molecules NHC1 and

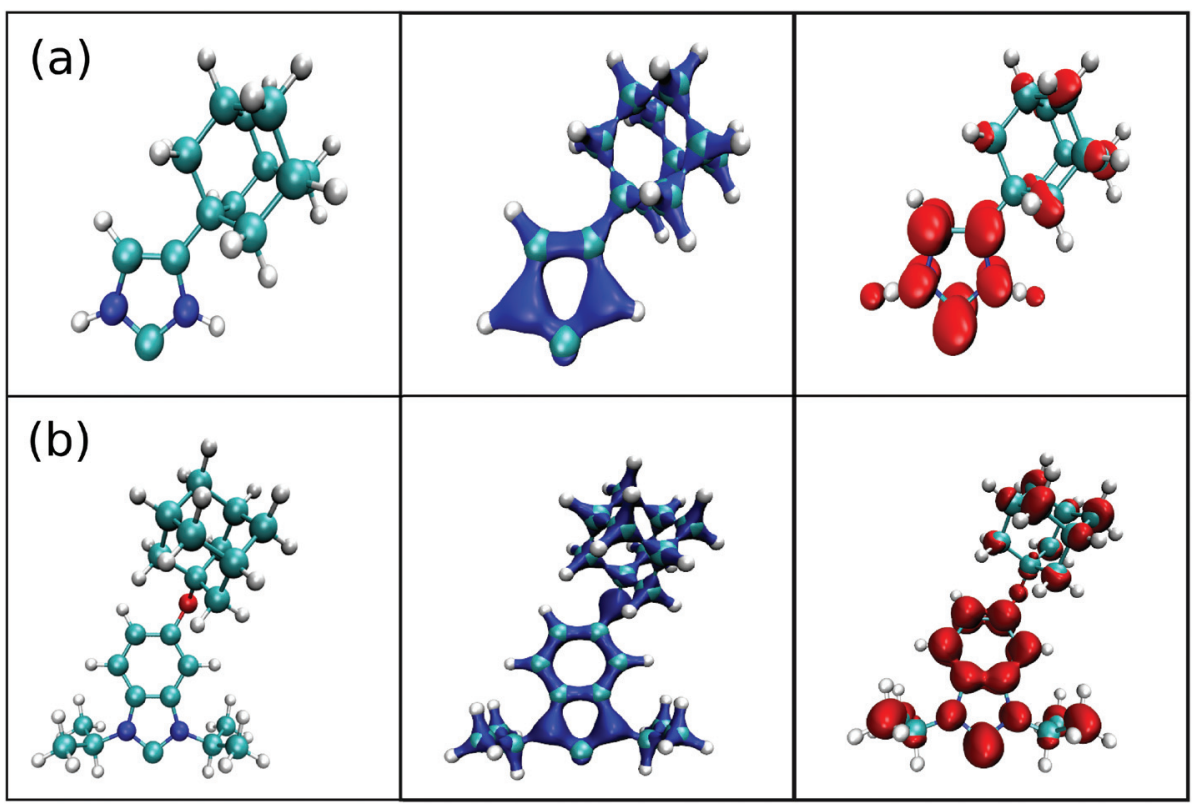

Fig. 1 (a) The NHC1-functionalized adamantane (left), the charge density of its highest occupied molecular orbital (HOMO) (middle; in blue), and the charge density of the lowest unoccupied molecular orbital (LUMO) (right; in red) are shown. (b) The NHC2-functionalized adamantane (left), the respective charge density of the HOMO (middle; in blue), and that for the LUMO (right; in red) are also given. 
NHC2. In the following sections, we refer to these functionalized diamondoids as NHC1-ada and NHC2-ada, respectively. The notation NHC-ada/metal (or modified metal surfaces) refers to the NHC-mediated adamantane SAMs on one of the metal surfaces.

\section{Structural analysis}

We begin the discussion with the analysis of the structural characteristics of all carbene-mediated diamondoid surfaces. All structurally relaxed SAMs are depicted in Fig. 2. The distance of the carbene-functionalized diamondoid from the surface is also denoted through arrows, which is calculated based on the bond-length of the lowest carbon atom of the carbene-functionalized diamondoid to the closest metal atom of the surface. We observe a variability in the ' $\mathrm{C}$-metal' bondlength for the different molecules and metal surfaces in the range of 1.97-2.28 $\AA$. An overall closer arrangement is evident for the diamondoid-SAMs on Pt(111), followed in trend by the diamondoid-SAMs on $\mathrm{Au}(111)$ evident due to the shorter bond (1.97 $\AA$ for the NHC1-ada case and 2.02 $\AA$ for the NHC2-ada case) of the functionalized diamondoid with the Pt surface. For the $\mathrm{Au}(111)$ surface, these values are $2.08 \AA$ for NHC1-ada and $2.12 \AA$ for NHC2-ada. As discussed in the literature, ${ }^{43}$ despite various NHC structures being available, the $\mathrm{C} / \mathrm{N}$ ratios for the films and nanoparticles investigated using X-ray photoelectron spectroscopy (XPS) were compared to previous experimental studies and were found to be optimal for the NHC with the $\mathrm{C}_{3} \mathrm{H}_{7}$ ligand. The NHC coverage for this case was also found to be dense enough to prevent other impurities from binding to other sites not covered by the NHC. This is the same molecule (NHC2) we chose in our investigations due to previous experimental evidence. As implied in the introduction, the family of NHC molecules can assume a number of variations, other than $\mathrm{C}_{3} \mathrm{H}_{7}$. Additional ligands have not been investigated here. The aim of this work was not an extensive study of such ligands and NHC members, rather to provide a proof of principles of the efficiency of such molecules in promoting the binding of diamondoids on metal surfaces in order to form self-assembled layers of diamondoids. The larger bond-length in both Ag SAMs is an indication of a less stable SAM, which will be further evaluated in the following sections. For the $\mathrm{Ag}$ surface, the respective bond-lengths are $2.19 \AA$ for NHC1-ada and $2.28 \AA$ for the NHC2-ada case. The shorter bonds of the diamondoid-SAMs on $\mathrm{Au}(111)$ and Pt(111) denote a stronger bonding compared to the Ag(111) surfaces.

In the case of NHC2-ada, two of the hydrogen atoms of the carbene molecule come close to the metal surfaces. Note that these $\mathrm{H}$ atoms are all bonded to secondary $\mathrm{C}$ atoms in the ligand. One of these secondary $\mathrm{C}$ atoms is bonded to the $\mathrm{N}$ atom of the carbene ring. The distance of the $\mathrm{H}$ atom bonded to the secondary $\mathrm{C}$ atom (close to the nitrogen) and the metal surface was found to be 2.86, 2.80, and $2.05 \AA$ for the $\mathrm{Au}, \mathrm{Ag}$, and Pt surfaces, respectively. In the case of $\mathrm{Pt}(111)$, these distances are quite small indicating that two of the hydrogen atoms attached to a secondary carbon atom of the carbene $\mathrm{C}_{3} \mathrm{H}_{7}$ ligands could also bind to the metal surface. This will be confirmed by the HOMO re-distribution analysis below. The distance of the $\mathrm{H}$ atoms bonded to the other two secondary $\mathrm{C}$ atoms in the ligand which is closest to the metal (a)

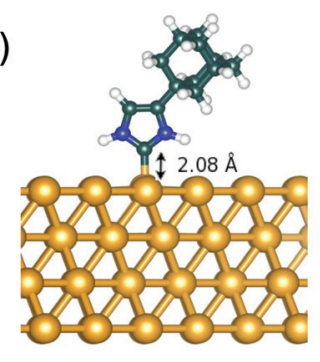

$\mathrm{Au}$

(d)

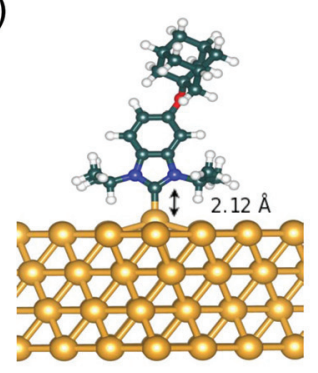

(b)

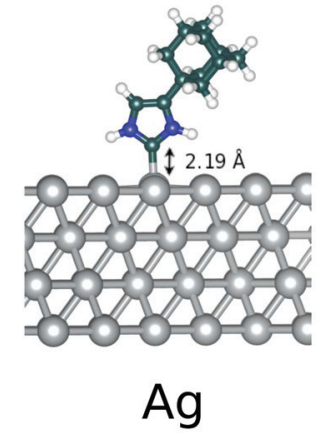

(e)

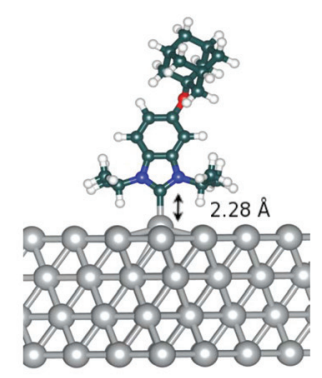

(c)

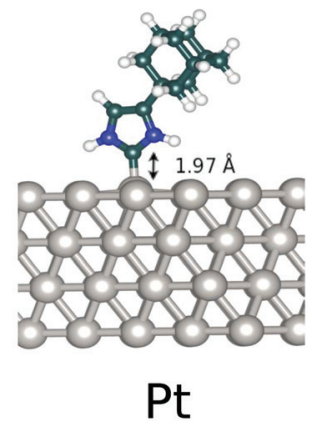

(f)

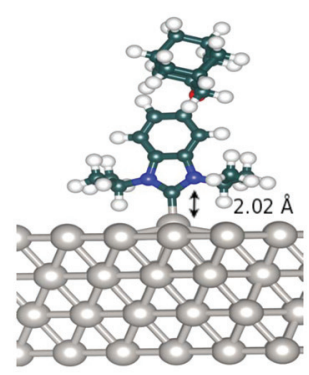

Fig. 2 (a)-(c) Adsorption of the NHC1-mediated adamantane SAMs on Au(111), Ag(111), and Pt(111), respectively. (d)-(f) Adsorption of the NHC2mediated adamantane SAMs on the same surfaces. The black arrows indicate the C-metal bond-lengths in each case. 
Table 1 Structural properties and adsorption energies $\left(E_{\text {ads }}\right)$ for the carbene-mediated diamondoid SAMs on metal surfaces depicted in Fig. 2. Energies are given in $\mathrm{eV}$, angles in degrees, and bond-lengths in $\AA$

\begin{tabular}{|c|c|c|c|c|c|c|c|c|c|c|c|c|}
\hline \multirow[b]{2}{*}{ SAMs } & \multicolumn{4}{|c|}{ Gold (111) } & \multicolumn{4}{|c|}{ Silver (111) } & \multicolumn{4}{|c|}{ Platinum (111) } \\
\hline & $E_{\text {ads }}$ & $\mathrm{C}-\mathrm{Au}$ & $\mathrm{C}-\mathrm{Au}-\mathrm{Au}$ & $\mathrm{H}-\mathrm{Au}$ & $E_{\text {ads }}$ & $\mathrm{C}-\mathrm{Ag}$ & $\mathrm{C}-\mathrm{Ag}-\mathrm{Ag}$ & $\mathrm{H}-\mathrm{Ag}$ & $E_{\text {ads }}$ & $\mathrm{C}-\mathrm{Pt}$ & $\mathrm{C}-\mathrm{Pt}-\mathrm{Pt}$ & $\mathrm{H}-\mathrm{Pt}$ \\
\hline NHC2-ada & -1.700 & 2.12 & 103 & 2.86 & -1.067 & 2.28 & 105 & 2.80 & -1.921 & 2.02 & 100 & 2.05 \\
\hline
\end{tabular}

is 3.18, 3.22 and $3.11 \AA$ for $\mathrm{H}-\mathrm{Au}, \mathrm{H}-\mathrm{Ag}$, and $\mathrm{H}-\mathrm{Pt}$, respectively. For the NHC1-ada case, the distances of the lowest hydrogen atoms of the carbene ring are 3.32, 3.16, and $2.75 \AA$ for $\mathrm{H}-\mathrm{Au}$, $\mathrm{H}-\mathrm{Ag}$ and $\mathrm{H}-\mathrm{Pt}$, respectively.

Another important structural detail is the relative orientation of the adsorbed NHC-adamantane with respect to the metal surfaces. A close inspection of all structures in Fig. 2 reveals that for each NHC-ada its vertical arrangement with respect to all three surfaces is almost the same. In order to prove this, we have calculated the angle between the carbene carbon atom which is closer to the surface (the one involved in the 'C-metal' bond mentioned above), the closest metal atom and one of its nearest neighbors. These angles lie in the range 93-96 ${ }^{\circ}$ for NHC1-ada and $100-106^{\circ}$ for $\mathrm{NCH} 2$-ada for all three surfaces. The values denote an orthonormal arrangement of the carbene-mediated adamantane-SAMs on the metal surfaces which is observed for both NHC1-ada and NHC2-ada. In the latter case, though, and for all three metal surfaces, the metal atom bonded to the carbene-functionalized adamantane was slightly pulled off the metal surface in order to form a stronger bond with the molecules. Hence, the respective angles are about $10^{\circ}$ larger than for NHC1-ada. This higher "attraction" of the closest metal atom from the NHC2-ada was also reported in a relevant experimental study. ${ }^{43}$ This behavior was not observed for the NHC1-ada case on any of the surfaces. The most important structural characteristics for the NHC-mediated adamantane-SAMs investigated here are summarized in Table 1.

\section{Adsorption energies}

In order to assess the quality of the SAM bonding on the metal surfaces, we have investigated the adsorption energies of the modified surfaces. The adsorption energy, $E_{\text {ads }}$, for a molecule adsorbed on a surface is the difference between the total energy of the molecule/metal system $\left(E_{\text {total }}\right)$ and the sum of the total energies of the two components. This sum includes the total energy of the clean metal surface $\left(E_{\text {clean }}\right)$ and the total energy of the isolated molecule $E_{\mathrm{mol}}$. The following equation holds for the adsorption energy:

$$
E_{\text {ads }}=E_{\text {total }}-E_{\text {clean }}-E_{\text {mol }}
$$

The adsorption energies for both NHC-ada on the gold and platinum surfaces are found in the range -1.70 to $-1.92 \mathrm{eV}$. Accordingly, no significant qualitative difference in their stability can be inferred for these cases.
For the silver surfaces, these energies are about 30-45\% higher compared to the NHC-ada adsorption on gold and platinum. In this respect, the SAMs can be easily formed on $\mathrm{Au}(111)$ and $\mathrm{Pt}(111)$ than on $\mathrm{Ag}(111)$. The fact that for the same metal surface, both SAMs lead to similar adsorption energies, can be understood as a similar efficiency of the metal surface in adsorbing both the NHC1- and the NHC2functionalized adamantane. This similarity is evident despite the fact that NHC1-ada does not contain the $\mathrm{C}_{3} \mathrm{H}_{7}$ ligand attached to nitrogen as in the NHC2-ada case. The stability of NHC2-ada has already been observed experimentally revealing the practical interest of NHC2-ada SAMs. ${ }^{43}$ Accordingly, due to the similarity in the adsorption energies, we can argue that the theoretical molecule NHC1-ada considered here could be an additional potential candidate in forming carbene-mediated SAMs on metal surfaces. All adsorption energies as calculated through eqn (1) are summarized in Table 1.

According to the above analysis, the bonding of the carbene-mediated diamondoid-SAMs on metal surfaces is stronger compared to thiol-mediated diamondoid-SAMs on the same surfaces. Specifically, our calculations lead to longer bonds in the latter case. For thiol-functionalized adamantane molecules, the $\mathrm{S}-\mathrm{Au}, \mathrm{S}-\mathrm{Ag}$, and $\mathrm{S}-\mathrm{Pt}$ bond-lengths (which correspond to the SAM-surface bonding) were found to be 2.56, 2.55, and $2.33 \AA$, respectively. The respective adsorption energies are $-2.16,-2.18$, and $-3.15 \mathrm{eV}$. At first sight, these denote a better adsorption of sulfur-bonded adamantane on the metal surfaces. For thiol, the preferred adsorption site on the metal surfaces is the fcc site, while for the NHC-ada the preferred adsorption site is the on-top site. This is confirmed by our initial calculations and experiments. ${ }^{44}$ According to recent theoretical investigations, DFT simulations with common GGA functionals incorrectly favor adsorption sites with a high coordination, such as the fcc site, rather than a low coordination site, such as the on top site. ${ }^{45}$ Note that though the fcc site for thiol-mediated adsorption is preferred in the experiments, ${ }^{44}$ the DFT-derived adsorption energies for the fcc site have not always been in good agreement with experiments. This was evident in the case of CO adsorption on Pt(111). ${ }^{46}$ Due to this possible discrepancy between experimental and theoretical data on the preferable binding sites, we focus on the comparison of bond-lengths rather than binding sites. Accordingly, we compare the bond-lengths between the S-mediated and NHC-mediated SAM adsorption on the metal surfaces. These bond-lengths are significantly shorter in the latter case, and differ about 11-19\% between the two cases. 
This finding indicates a stronger bonding of the NHCdiamondoid SAMs than thiol-diamondoid SAMs on the metal substrates.

\section{Electronic properties - projected density of states (PDOS)}

Isolated NHC-functionalized adamantane. For the analysis of the electronic properties of the structures studied here, we begin with the projected electronic density of states (PDOS) and the frontier orbitals of the free-standing (no metal surface) carbene-functionalized adamantane. The latter are the highest occupied and lowest unoccupied molecular orbitals, HOMO and LUMO, respectively. The charge density of the frontier orbitals of the free standing SAMs is sketched in Fig. 1. We observe that the charge density for the HOMO is quite delocalized over the whole molecule, including the adamantane and NHC parts (for both NHC1 and NHC2). On the other hand, the charge density for the LUMO is more localized on the carbene ring and the lower $\mathrm{N}-\mathrm{C}-\mathrm{N}$ atoms, the tip of the carbene ligand. This ligand promotes the binding of the molecule to the metal surface. These features differ from the ideal adamantane case, as expected. For the ideal adamantane, the charge density for the HOMO is localized in between the carbon-hydrogen bonds and the charge density for the LUMO is delocalized and spread out over the molecule's surface. ${ }^{47}$

The respective PDOS of the isolated NHC-ada molecules in the left panel of Fig. 3 are considerably altered by the presence of the carbene as compared to the ideal adamantane. ${ }^{47}$ For both molecules, the s-orbitals contribute more to the electronic density of states in both the HOMO and the LUMO levels. A comparison of both carbene variations, NHC1 and NHC2, reveals that the s- and p-distributions in the PDOS are similarly broad. The functionalization introduces electronic states in the band-gap of the ideal adamantane, decreasing in this way the electronic band-gap of carbene-functionalized adamantane. This band-gap reaches $3.62 \mathrm{eV}$ for NHC1-ada and
$3.37 \mathrm{eV}$ for NHC2-ada which is a decrease of almost $50 \%$ compared to the value for ideal adamantane. ${ }^{47}$ Note that the results for the electronic band-gaps were obtained with DFT without any correction for the band-gap calculation. Accordingly, these values are expected to be off with respect to the experimental values. However, our simulations can capture the trends in the modification of the electronic band-gap due to the carbene-functionalization and this trend is of interest here.

Adamantane SAM modified metal surfaces. The adsorption of the NHC-ada molecules on the metal surfaces alters the electronic properties of the clean surfaces and the isolated NHC-ada. The electronic features of the adsorbed SAMs mapped through the PDOS are shown in the right panel of Fig. 3 and reveal the qualitative influence of the SAM adsorption on the surfaces. For all substrates, we observe that the HOMO of the molecule is slightly shifted towards the Fermi energy which indicates the bonding to the surface. The occupied levels become more spread out. The unoccupied levels become less peaked and slightly broader for the adsorbed molecules. The trend in the height of the peaks in the p-orbital contribution for the occupied electronic levels is altered in the adsorbed cases, as the third peak before the Fermi energy is more pronounced compared to the fourth peak in the non-adsorbed cases. This again denotes the tendency to 'move' the electronic levels in order to promote binding to the surface. According to the total density of states (not shown here), all occupied electronic levels enter the bandgap region of the free standing carbene-functionalized adamantane before adsorption.

In order to reveal the binding characteristics and the reactivity of the metal surfaces, we calculated the PDOS of the first layer of the metal substrates and the PDOS of the carbon atom of the carbene ring which is the closest and binds to the surface. For a better qualitative understanding of the nature of bonding and the hybridization of the electrons the
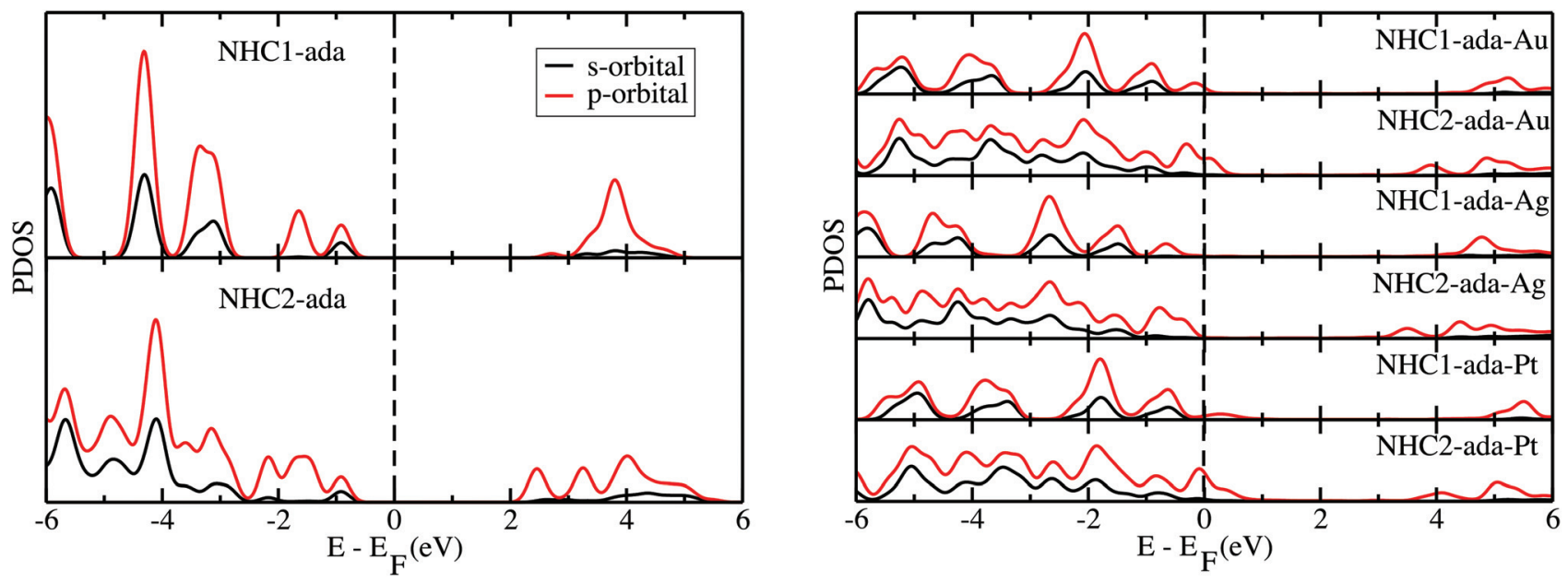

Fig. 3 Projected density of states (PDOS) for both carbene-functionalized adamantane molecules before (left) and after (right) adsorption. The legends indicate the molecule (left) and the surface on which it is adsorbed (right). The contributions from the s-and p-orbitals are depicted in black and red, respectively. The Fermi energy in each case was shifted to $0 \mathrm{eV}$. 


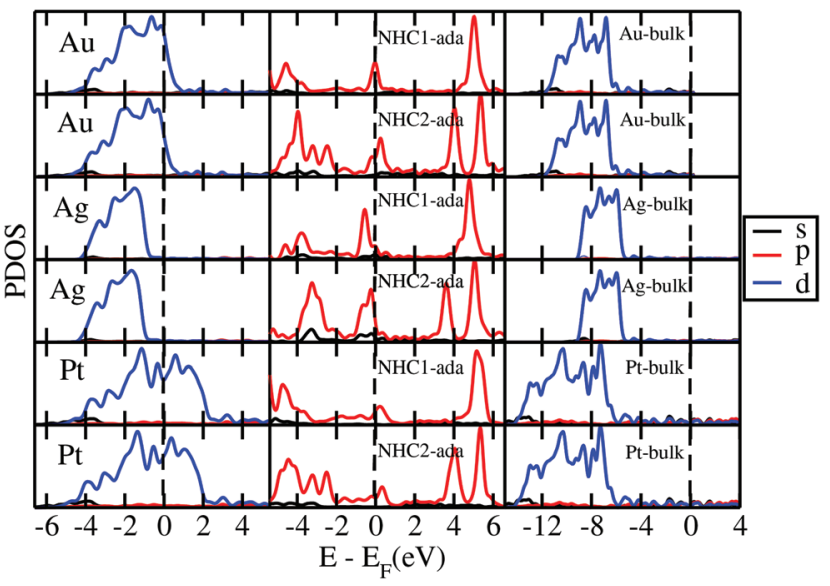

Fig. 4 The contributions of the s-, p-, and d-orbitals to the PDOS of the metal surfaces are depicted for the first layer of the metal surface for $A u(111), A g(111)$, and $\mathrm{Pt}(111)$ on the left. For the same surfaces, the middle graphs depict the PDOS contribution of the $s$ - and p-orbitals of the carbene carbon atom which is the closest and binds to the metal surface. The right panels depict the PDOS for the bulk metals before adsorption. The Fermi energy has been shifted to $0 \mathrm{eV}$ in all cases. The contributions of the s-, p-, and d-orbitals are shown in black, red, and blue, respectively.

contributions with respect to the angular momentum (s-, p-, and d-orbitals) in the PDOS are plotted in Fig. 4. A covalent bond between the molecule and the surface can be formed when d-orbitals contribute more to the density of states near the Fermi energy. In such a case, the p-electrons of the molecules can coordinate with the hybrid orbitals of the metal surface and promote bonding of the two structures.

Inspection of the left panels in this figure reveals that the contribution of the d-orbitals in the PDOS for the first layer of the $\mathrm{Au}$ and Pt surfaces at the Fermi level is very large compared to the respective contributions of the s- and p-orbitals. The contributions of the carbene carbon atom in the PDOS (right graphs in each panel of Fig. 4) reveal a peak in the p-orbital contributions around the Fermi energy. Accordingly, the p-orbitals of the lowest carbene atom are available to overlap with the d-orbitals of the metal and share electrons to form bonds. The overlapping is not so evident in $\mathrm{Ag}(111)$; a difference possibly assigned to its higher reactivity compared to gold and platinum. Since the p-orbital contribution to the PDOS for the NHC1-ada/Au and the NHC2-ada/Pt is more peaked at the Fermi level, it can be inferred that the bonding in these two cases should be slightly stronger compared to NHC2-ada/Au and NHC1-ada/Pt. For the latter structures, the p-orbital contributions of the carbene carbon atom are slightly off the Fermi level. This is in agreement with the shorter C-Au bond for NHC1-ada/Au compared to NHC2-ada/Au in Table 1. For $\operatorname{Pt}(111)$, though, we see a reverse behavior: the p-orbital contribution for NHC2-ada/Pt is peaked exactly at the Fermi level, but the C-Pt bond according to Table 1 is $2 \%$ longer than in NHC1-ada/Pt for which the p-orbital contribution in the PDOS shows a peak slightly off the Fermi level. Neverthe- less, the adsorption energy for NHC2-ada/Pt is $4 \%$ higher than in NHC1-ada/Pt, denoting stronger adsorption in the former case. A possible explanation lies on the existence of the $\mathrm{C}_{3} \mathrm{H}_{7}$ ligand in NHC2-ada and the attraction of the nearest hydrogen atoms on $\mathrm{Pt}(111)$ as will be revealed through the analysis of the surface morphologies in the following section.

The calculated dipole moments of the NHC-ada/metal structures were extracted from our calculations. The $x$ - and $y$-coordinates of the dipole moments of all six structures were zero. Only the $z$-component of the dipole moments was found to be non-zero, and for the NHC1-ada/metal on $\mathrm{Au}(111) \mathrm{Ag}$ (111), and $\operatorname{Pt}(111)$ are $-6.532,-5.519$, and -6.340 Debye, respectively. For NHC2/ada on the same metals, the dipole moment values are $-7.224,-5.735$, and -6.912 Debye, respectively. Note that the $z$-axis was taken to be normal to the modified surfaces. All dipole moments are negative indicating that in all cases, the dipole moment vector points from the adsorbed molecule towards the metal surface. Overall, the modified gold substrates show higher dipole moments. The NHC2-mediated adamantane SAM also corresponds to a larger dipole moment compared to the NHC1-ada adsorbed structures.

\section{Work function}

As a measure of the efficiency of carbene-mediated adamantane SAMs on metal surfaces for practical applications, we performed the analysis of their work functions $\Phi$. We expect these to be modulated with respect to the work function of the clean metal surfaces, in a way that charge transfer from the modified surfaces becomes more efficient. This feature would be valuable for potential electronics applications of the NHC-ada/ metals. An adsorbate layer contributes to the charge transfer in the adsorption procedure, significantly modulating the work function of the metal surface. The electrons leaving the metal surface will have to pass through the interface dipole layer. The orientation of the dipole influences the efficiency in the removal of electrons. The electrostatic potential at each point of the unit cell in our simulations is used to create an electrostatic profile along the $z$-direction normal to the surface. The work function is obtained from the electrostatic profile value in the vacuum area. The relationship of the work function and the surface dipole moment is given by:

$$
\Delta \Phi=-\frac{e}{\varepsilon_{0}}\left(\mu_{z}-\mu_{z, 0}\right)=-\frac{e}{\varepsilon_{0}} \Delta \mu
$$

where $\mu_{0}$ is the surface dipole per unit area, $\mu_{z}$ is the surfacenormal dipole per unit area for the SAM adsorbed surface, $e$ is the charge, and $\varepsilon_{0}$ is the dielectric constant. A positive value of $\mu$ indicates a dipole that is pointing away from the bulk, which leads to a decrease of the work function. A negative value of $\mu$ corresponds to a dipole pointing to the bulk, thus increasing the work function and hindering the charge transfer.

The calculated work functions of the clean $\mathrm{Au}(111)$, $\mathrm{Ag}(111)$, and $\mathrm{Pt}(111)$ surfaces according to our calculations are $5.25,4.5$, and $5.73 \mathrm{eV}$, respectively. We have found that the adsorption of the NHC-ada SAMs lowers the work function 
considerably. To assess the two contributions from the molecule and the metal surface, we must consider two additional systems. We first remove the molecular layer and compute the electrostatic profile of the relaxed free gold surface. As a second step, we remove the gold surface and compute the electrostatic profile of the free radical SAM. We then superpose

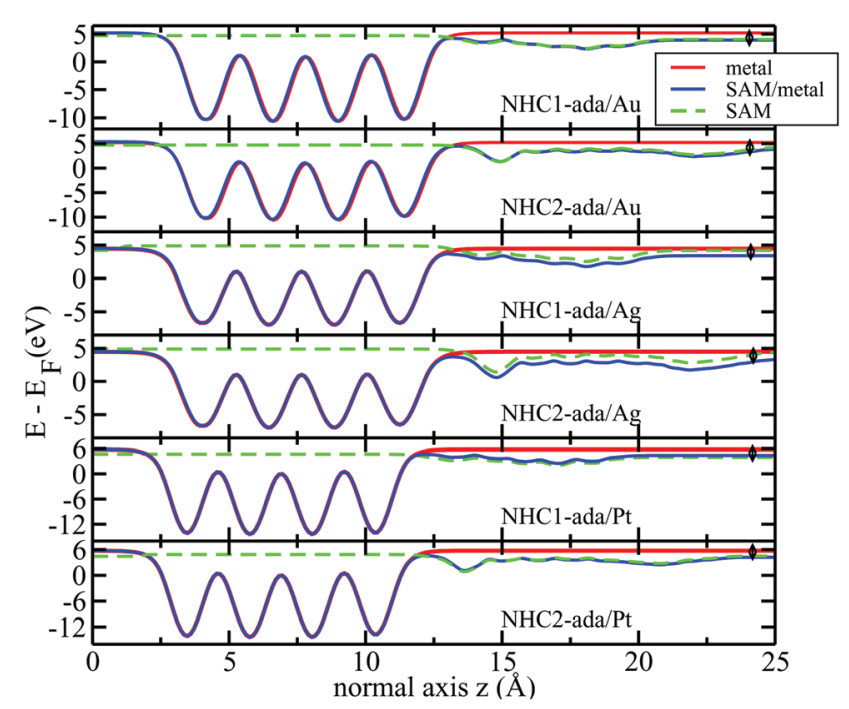

Fig. 5 Plane averaged electrostatic profiles of the two different configurations of carbene-functionalized adamantane SAMs on all three surfaces. The labels denote the specific modified surface for each panel. The zero energy is set at the Fermi level of each system. In blue, green, and red are shown the electrostatic potentials of the bare metal substrate, the free molecule SAM, and the whole system, respectively. The black arrows between the red and the blue lines point to the work function shifts after adsorption. the electrostatic profile of these two systems after aligning the vacuum level on the right part as shown in Fig. 5. In this figure, the modulation of the work function due to the adsorption of the carbene-mediated adamantane monolayers is reported. The work function of the metal surface modified with the NHC1-functionalized adamantane was found to be $3.80 \mathrm{eV}$ for $\mathrm{Au}(111), 3.26 \mathrm{eV}$ for $\mathrm{Ag}(111)$, and $4.18 \mathrm{eV}$ for $\mathrm{Pt}(111)$. For the NHC2-ada molecule, the work function was $3.82 \mathrm{eV}$ for $\mathrm{Au}(111), 3.30 \mathrm{eV}$ for $\mathrm{Ag}(111)$, and $4.30 \mathrm{eV}$ for $\mathrm{Pt}(111)$. For both SAMs, we find a significant decrease of the work function of the order $37-38 \%$ for $\mathrm{Au}(111), 36-38 \%$ for $\mathrm{Ag}(111)$, and $33-37 \%$ for $\mathrm{Pt}(111)$. Overall, the work functions for both SAMs and all three surfaces are very similar and differ only about $1-3 \%$. For the NHC2-ada/metals the work functions are slightly higher and can presumably be assigned to the presence of the additional two $\mathrm{C}_{3} \mathrm{H}_{7}$ ligands in NHC2.

\section{Surface morphology}

In order to reveal the electronic and structural morphologies of the metal surfaces, we looked into two additional features, the localization of the highest occupied orbitals close to the surface and the simulated scanning tunneling microscopy (STM) images. We first inspect panels (a)-(c) and (g)-(i) of Fig. 6. In these, the charge density distributions for the HOMO after adsorption reveal the re-arrangement of the total electronic charge due to the adsorption of the NHC-ada SAMs on the metal substrates. From this figure it is clearly evident that the occupied orbitals after adsorption are mostly localized next to the bonding sites of the carbene lower tip and the surface of the metal. Qualitatively, no significant differences in the orbitals for the Au and Ag modified surfaces were found. Nevertheless, the orbitals tend to occupy the upper part of the carbene (a)

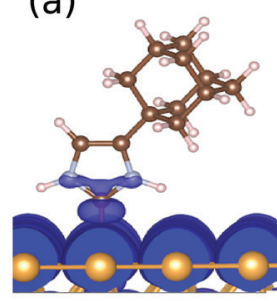

(g)

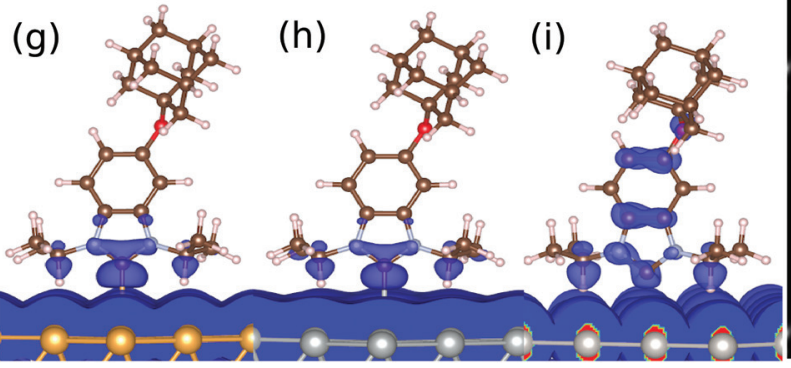

(c)

(b)
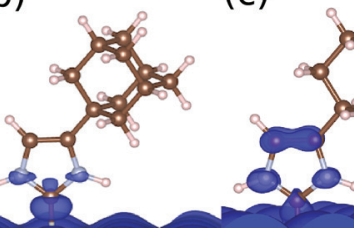
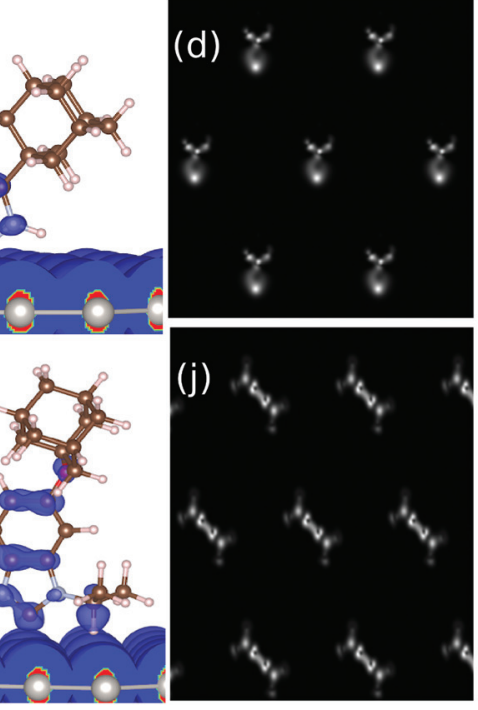
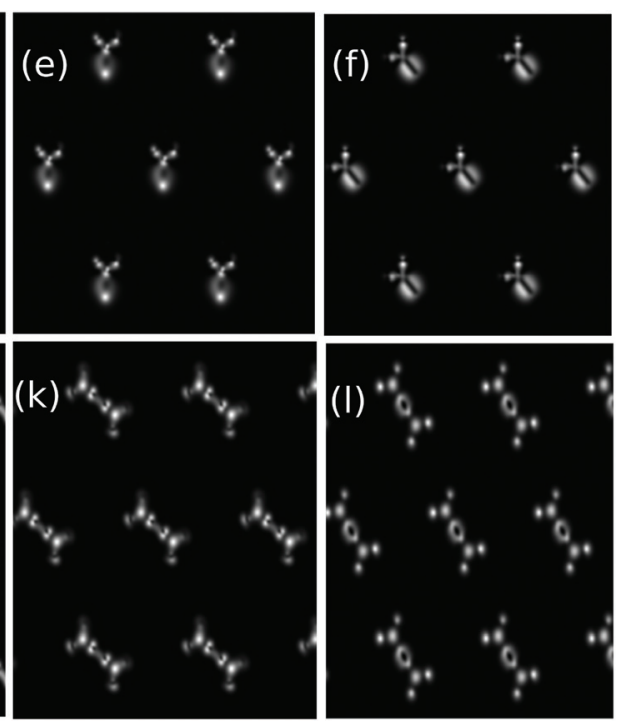

Fig. 6 In (a)-(c) the re-distribution of the charge density of the HOMO after adsorption of NHC1 on the metal surfaces Au(111), Ag(111), and Pt(111), respectively is shown. In (d)-(f) the simulated STM images of the systems in (a)-(c) are also presented. Panels (g)-(i) depict the charge re-distribution of the HOMO after adsorption of NHC2 on Au(111), $\mathrm{Ag}(111)$, and Pt(111), respectively. Panels (j)-(l) correspond to the simulated STM images of the systems in (g)-(i). For the STM simulations a negative bias of $-1 \mathrm{~V}$ was applied to the surface at a height of $10 \AA$ above the surface. 
Table 2 Bader charge analysis comparing the charges on the SAM and the metal surfaces before and after adsorption. The first line denotes the modified system. All values are given in electrons

\begin{tabular}{llllllll}
\hline & \multicolumn{2}{l}{ NHC1-ada } & & & \multicolumn{2}{l}{ NHC2-ada } \\
\cline { 2 - 3 } \cline { 7 - 8 } & $\mathrm{Au}$ & $\mathrm{Ag}$ & $\mathrm{Pt}$ & & $\mathrm{Au}$ & $\mathrm{Ag}$ & $\mathrm{Pt}$ \\
\hline NHC-ada & +0.47 & +0.31 & +0.52 & & +0.48 & +0.26 & +0.51 \\
metal & -0.28 & -0.10 & -0.34 & & -0.25 & -0.03 & -0.31
\end{tabular}

ring close to the adamantane in the case of the modified $\mathrm{Pt}(111)$. We also observe that the charge in the case of $\mathrm{Pt}(111)$ is spread over the whole carbene ring, which is not the case in the modified $\mathrm{Au}(111)$ and $\mathrm{Ag}(111)$ surfaces. In the latter two cases, the charge re-arrangement occurs mainly around the carbene atoms which are closer to the metal surface and are involved in the bonding.

A Bader charge analysis was performed in order to reveal the effect of SAM adsorption on the modified surfaces. ${ }^{48}$ Specifically, we calculate the change in the total charge of the molecule and the surface after the adsorption of the molecule on the metal surfaces, compared to its total charge before adsorption. All results are summarized in Table 2. The changes in the total charge due to adsorption are given separately for the adsorbed molecule only ('NHC-ada' in the table) and the substrate only ('metal' in the table). Overall, the surfaces have a negative change in their charge after adsorption, while the NHC-ada molecules have a positive change. This finding indicates that the charge re-arrangement in the NHCada/metal structures is being done in a way to move the charge towards the molecule, an important property towards applications like field-emission displays.

STM images. The application of a bias voltage between the tip and the NHC-ada/metal when these are brought close to each other at a distance of a few angstroms, will enable the electrons to tunnel through the vacuum space between the two surfaces. The electron flow is usually referred to as tunneling current and is dependent on three parameters: the distance of vacuum between the surfaces, the applied bias voltage, and the local density of states. We keep the distance of an STM tip at about $10 \AA$ above the NHC-ada/metal surface along the normal $z$-axis, while scanning with the tip the $x y$-plane of this surface to produce a constant current. We consider a negative surface bias of $-1 \mathrm{~V}$ in order to sample all occupied surface states from this energy value up to the Fermi level. This enables the measurement of the topography of the NHC-ada SAM surfaces and reveals the STM images of all the modified metal surfaces studied here.

All six right panels of Fig. 6 show the simulated STM images of all SAM-modified metal surfaces. These images are produced by calculating the contour plots of the tunneling current density on the plane parallel to the surface. From this analysis, distinct bright spots corresponding to the carbenefunctionalized adamantane adsorbed onto the surface are seen. A brighter spot is observed in all STM panels on the atoms which are more involved in the bonding to the surface. A similar behaviour to the charge re-distributions (left panels of the same figure) is evident in terms of the molecular orientation of the carbene-functionalized adamantane on the metal substrate. The more extensive structure of the NHC2-ada is clear in panels $(\mathrm{j})-(\mathrm{l})$. This feature is based on the additional $\mathrm{C}_{3} \mathrm{H}_{7}$ ligand in NHC2. A slightly different orientation of both NHC1-ada and NHC2-ada on Pt(111) compared to their orientation on the gold and silver surfaces is evident. Note that before structural relaxation all NHC-ada SAMs were placed with the same orientation on all metal surfaces. Small differences in the bright spots in the case of $\mathrm{Pt}(111)$ also denote different bonding characteristics compared to the $\mathrm{Au}(111)$ and $\mathrm{Ag}(111)$ cases. This is in agreement with the observation we made in Fig. 6(c) and (i), that the charge on $\mathrm{Pt}(111)$ involves the whole carbene ring and not only the lower $\mathrm{N}-\mathrm{C}-\mathrm{N}$ atoms closer to the surface as in the modified gold and silver surfaces. The different charge re-distribution of the molecule on $\mathrm{Pt}(111)$ is also clear by comparing panel (i) to panels (g) and (h) of Fig. 6. The shorter distance of the H atoms of the NHC2 ligand to the $\mathrm{Pt}(111)$ surface is given in Table 1 , is linked to a bonding of these $\mathrm{H}$ atoms to the Pt surface. The adsorbate (NHC2-ada) orientation on $\mathrm{Pt}(111)$ changes in order to facilitate this bonding. This is not the case in NHC1-ada/Pt for which we also observe a different orientation with respect to NHC1-ada/Au and NHC1-ada/Ag. The difference here is based on the observation in Fig. 6(c) that the charge re-distribution in NHC1-ada/Pt involves the whole carbene ring. Accordingly, the charge of the whole ring promotes (or is involved in) the bonding and again the NHC1-ada needs to re-orient to account for this additional attraction.

\section{Conclusions}

In summary, using quantum-mechanical simulations we have modeled the adsorption of carbene-functionalized diamondoids on metal surfaces. Overall, $\mathrm{Au}(111)$ and $\operatorname{Pt}(111)$ were found to be the most efficient metal surfaces for adsorbing carbene-mediated diamondoid SAMs. Orbitals of the d-symmetry are essential in order to have orbital overlapping with carbon p-electrons and bond formation as in the case of the $\mathrm{Au}(111)$ and $\mathrm{Pt}(111)$ surfaces. In $\mathrm{Ag}(111)$, the weaker bonding was justified by the non-availability of the d-orbital of the metallic first layer of the surface around the Fermi level. Calculation of the work functions of carbene-mediated diamondoid SAMs on $\mathrm{Au}(111), \mathrm{Ag}(111)$, and Pt(111) surfaces demonstrated that the modulation in the work function with respect to the properties of clean metal surfaces and a dynamic tuning can be easily achieved in NHC-ada/metal devices for organic electronic applications. The charge re-distribution and the simulated STM images of the surfaces showed that on Pt(111) the whole carbene ring of the NHC-ada molecule is associated with the SAM/metal bonding, increasing the adsorption energy of SAMs. By comparing the molecular adsorption energies on reconstructed $\mathrm{Au}(111), \mathrm{Ag}(111)$, and $\mathrm{Pt}(111)$ surfaces, we 
conclude that these geometries are stable and of practical interest. However, it is essential to examine the thermal stability of these structures and the influence of environmental conditions on their stability. Nevertheless, carbene molecules have proven to be a better alternative to the thiol-based bonding of diamondoids on metallic surfaces, opening up the path for more stable and efficient SAM/metal devices. Based on these findings and in view of electronics applications such as field emitting devices or sensors, optical properties of carbenemediated SAMs on metal surfaces need to be investigated. Work along these lines is ongoing.

\section{Acknowledgements}

B. A. wishes to thank $\mathrm{H}$. Fu for her kind help in the initial phase of this project, the Institute of Physics at the Chinese Academy of Sciences for its kind hospitality and for granting use of its computational resources. B. A. and M. F. acknowledge support from the Juniorprofessorenprogramm BadenWürtemberg (MWK) and the collaborativenetwork SFB 716 "Dynamic simulations of systems with large particle numbers" funded by the German Funding Agency (Deutsche Forschungsgemeinschaft-DFG). S. M. acknowledges partial financial support from MOST (grant 2015CB921001) and NSFC (grant 11474328 and 11222431). This research was supported in part by the bwHPC initiative and the bwHPC-C5 project provided through associated compute services of the JUSTUS HPC facility at the University of Ulm. The bwHPC and bwHPC-C5 (http://www.bwhpc-c5.de) are funded by the Ministry of Science, Research and the Arts Baden-Württemberg (MWK) and the German Research Foundation (DFG). Part of this work was performed on the computational resource for HLR Phase I funded by the Ministry of Science, Research and the Arts Baden-Württemberg and DFG.

\section{References}

1 A. T. Balaban and P. V. R. Schleyer, Tetrahedron, 1978, 34, 3599-3609.

2 J. E. Dahl, S. G. Liu and R. M. K. Carlson, Science, 2003, 299, 96-99.

3 H. Schwertfeger, A. Fokin and P. Schreiner, Angew. Chem., Int. Ed., 2008, 47, 1022-1036.

4 Y. Lifshitz, T. Köhler, T. Frauenheim, I. Guzmann, A. Hoffman, R. Q. Zhang, X. T. Zhou and S. T. Lee, Science, 2002, 297, 1531-1533.

5 W. L. Yang, et al., Science, 2007, 316, 1460-1462.

6 Y. Wang, E. Kioupakis, X. Lu, D. Wegner, R. Yamachika, J. E. Dahl, R. M. K. Carlson, S. G. Louie and M. F. Crommie, Nat. Mater., 2008, 7, 38-42.

7 G. Zhang, Phys. Today, 2013, 66(3), 59.

8 G. C. McIntosh, M. Yoon, S. Berber and D. Tománek, Phys. Rev. B: Condens. Matter, 2004, 70, 045401.

9 Y. Xue and G. A. Mansoori, Int. J. Mol. Sci., 2010, 11, 288.
10 M. Yao, P. Stenmark, E. Abou-Hamad, F. Nitze, J. Qin, C. Goze-Bac and T. Wågberg, Carbon, 2011, 49, 11591166.

11 J. Zhang, Y. Feng, H. Ishiwata, Y. Miyata, R. Kitaura, J. E. P. Dahl, R. M. K. Carlson, H. Shinohara and D. Tománek, ACS Nano, 2012, 6, 8674-8683.

12 S. B. Legoas, R. P. B. dos Santos, K. S. Troche, V. R. Coluci and D. S. Galvão, Nanotechnology, 2011, 22, 315708.

13 L. Landt, C. Bostedt, D. Wolter, T. Möller, J. E. P. Dahl, R. M. K. Carlson, B. A. Tkachenko, A. A. Fokin, P. R. Schreiner, A. Kulesza, R. Mitrić and V. BonačićKoutecký, J. Chem. Phys., 2010, 132, 144305.

14 L. Landt, M. Staiger, D. Wolter, K. Klünder, P. Zimmermann, T. M. Willey, T. van Buuren, D. Brehmer, P. R. Schreiner, B. A. Tkachenko, A. A. Fokin, T. Möller and C. Bostedt, J. Chem. Phys., 2010, 132, 024710.

15 S. Roth, D. Leuenberger, J. Osterwalder, J. Dahl, R. Carlson, B. Tkachenko, A. Fokin, P. Schreiner and M. Hengsberger, Chem. Phys. Lett., 2010, 495, 102-108.

16 N. D. Drummond, A. J. Williamson, R. J. Needs and G. Galli, Phys. Rev. Lett., 2005, 95, 096801.

17 U. Drechsler, B. Erdogan and V. M. Rotello, Chem. - Eur. J., 2004, 10, 5570-5579.

18 B. D. Gates, Q. Xu, M. Stewart, D. Ryan, C. G. Willson and G. M. Whitesides, Chem. Rev., 2005, 105, 1171-1196.

19 R. G. Nuzzo and D. L. Allara, J. Am. Chem. Soc., 1983, 105, 4481-4483.

20 J. C. Love, L. A. Estroff, J. K. Kriebel, R. G. Nuzzo and G. M. Whitesides, Chem. Rev., 2005, 105, 1103-1170.

21 C. D. Bain, E. B. Troughton, Y. T. Tao, J. Evall, G. M. Whitesides and R. G. Nuzzo, J. Am. Chem. Soc., 1989, 111, 321-335.

22 A. D. Jewell, H. L. Tierney and E. C. H. Sykes, Phys. Rev. B: Condens. Matter, 2010, 82, 205401.

23 D. V. Leff, L. Brandt and J. R. Heath, Langmuir, 1996, 12, 4723-4730.

24 D. I. Gittins and F. Caruso, Angew. Chem., Int. Ed., 2001, 40, 3001-3004.

25 C. Vericat, M. E. Vela, G. Benitez, P. Carro and R. C. Salvarezza, Chem. Soc. Rev., 2010, 39, 18051834.

26 J. J. Gooding and S. Ciampi, Chem. Soc. Rev., 2011, 40, $2704-2718$.

27 J. Noh, H. S. Kato, M. Kawai and M. Hara, J. Phys. Chem. B, 2006, 110, 2793-2797.

28 L. Srisombat, A. C. Jamison and T. R. Lee, Colloids Surf., A, 2011, 390, 1-19.

29 C. Vericat, G. A. Benitez, D. E. Grumelli, M. E. Vela and R. C. Salvarezza, J. Phys.: Condens. Matter, 2008, 20, 184004.

30 A. J. Arduengo, R. L. Harlow and M. Kline, J. Am. Chem. Soc., 1991, 113, 361-363.

31 O. Schuster, L. Yang, H. G. Raubenheimer and M. Albrecht, Chem. Rev., 2009, 109, 3445-3478.

32 J. C. Y. Lin, R. T. W. Huang, C. S. Lee, A. Bhattacharyya, W. S. Hwang and I. J. B. Lin, Chem. Rev., 2009, 109, 35613598. 
33 M. Niehues, G. Erker, G. Kehr, A. R. F. H. Pia Schwab, O. Blacque and H. Berke, Organometallics, 2002, 21, 29052911.

34 J. P. Wagner and P. R. Schreiner, J. Chem. Theory Comput., 2016, 12(1), 231-237.

35 G. Kresse and J. Furthmüller, Phys. Rev. B: Condens. Matter, 1996, 54, 11169-11186.

36 G. Kresse and D. Joubert, Phys. Rev. B: Condens. Matter, 1999, 59, 1758-1775.

37 P. E. Blöchl, Phys. Rev. B: Condens. Matter, 1994, 50, 1795317979.

38 J. P. Perdew, J. A. Chevary, S. H. Vosko, K. A. Jackson, M. R. Pederson, D. J. Singh and C. Fiolhais, Phys. Rev. B: Condens. Matter, 1992, 46, 6671-6687.

39 H. J. Monkhorst and J. D. Pack, Phys. Rev. B: Solid State, 1976, 13, 5188-5192.

40 M. Methfessel and A. T. Paxton, Phys. Rev. B: Condens. Matter, 1989, 40, 3616-3621.
41 P. E. Blöchl, O. Jepsen and O. K. Andersen, Phys. Rev. B: Condens. Matter, 1994, 49, 16223-16233.

42 W. P. Davey, Phys. Rev., 1925, 25, 753-761.

43 C. M. Crudden, J. H. Horton, I. I. Ebralidze, O. V. Zenkina, A. B. McLean, B. Drevniok, Z. She, H.-B. Kraatz, N. J. Mosey, T. Seki, E. C. Keske, J. D. Leake, A. RousinaWebb and G. Wu, Nat. Chem., 2014, 6, 409-414.

44 R. G. Nuzzo, B. R. Zegarski and L. H. Dubois, J. Am. Chem. Soc., 1987, 109, 733-740.

45 D. Otálvaro, T. Veening and G. Brocks, J. Phys. Chem. C, 2012, 116, 7826-7837.

46 P. J. Feibelman, B. Hammer, J. K. Nørskov, F. Wagner, M. Scheffler, R. Stumpf, R. Watwe and J. Dumesic, J. Phys. Chem. B, 2001, 105, 4018-4025.

47 B. Adhikari and M. Fyta, Nanotechnology, 2015, 26, 035701.

48 G. Henkelman, A. Arnaldsson and H. Jónsson, Comput. Mater. Sci., 2006, 36, 354-360. 